\section{BASECAMP-1: AN OBSERVATIONAL STUDY TO IDENTIFY RELAPSED SOLID TUMOR PATIENTS WITH HUMAN LEUKOCYTE ANTIGEN (HLA) LOSS OF HETEROZYGOSITY (LOH) AND LEUKAPHERESIS FOR FUTURE CAR T-CELL THERAPY}

${ }^{1}$ Julian Molina*, ${ }^{2}$ William Go, ${ }^{3}$ Scott Kopetz, ${ }^{4}$ Diane Simeone, ${ }^{5}$ Sandip Patel, ${ }^{1} Y i$ Lin, ${ }^{2}$ Kirstin Liechty, ${ }^{2}$ Michelle Fan-Port, ${ }^{6}$ Jason Perera, ${ }^{2}$ Armen Mardiros, ${ }^{7}$ Karl Beutner, ${ }^{7}$ Ariane Lozac'hmeur, ${ }^{2}$ Eric Ng, ${ }^{8}$ David Maloney, ${ }^{9}$ J Randolph Hecht. 'Mayo Clinic, Rochestser, MN, USA; ${ }^{2}$ A2 Biotherapeutics, Inc., Agoura Hills, CA, USA; ${ }^{3}$ University of Texas MD Anderson Cancer, Houston, TX, USA; ${ }^{4}$ New York University Langone Health, New York, NY, USA; ${ }^{5}$ University of California San Diego, La Jolla, CA, USA; ${ }^{6}$ former Tempus, Chicago, IL, USA; ${ }^{7}$ Tempus, Chicago, IL, USA; ${ }^{8}$ Fred Hutchinson Cancer Research Center, Seattle, WA, USA; ${ }^{9}$ David Geffen School of Medicine at UCLA, Los Angeles, USA

Background Solid tumors comprise $>90 \%$ of cancers. Metastatic colorectal cancer, non-small cell lung cancer, and pancreatic cancer are among the leading causes of cancer-related mortality (5-year overall survival: 14\%, 6\%, and 3\%, respectively). ${ }^{1}$ Chimeric antigen receptor (CAR) T-cell therapy demonstrated clinical outcomes in hematologic malignancies. ${ }^{2} 3$ However, translating engineered T-cell therapies to solid tumors proves difficult due to a lack of tumor-specific targets that discriminate cancer cells from normal cells. In previous studies, the use of a carcinoembryonic antigen T-cell receptors and mesothelin CARs both resulted in dose-limiting on-target, off-tumor toxicities. ${ }^{4} \mathrm{Tmod}^{\mathrm{TM}}$ CAR T-cell therapy addresses these challenges by leveraging dual receptors to create a robust AND NOT signal integrator capable of killing tumor cells, while leaving healthy cells intact (figure 1). ${ }^{6}$ Tmod platform technology is a versatile system that may be applied to $\mathrm{T}$ cells and natural killer cells in autologous and allogeneic settings.HLA LOH offers a definitive tumor versus normal discriminator target for CAR T-cell therapy. ${ }^{6} 7$ The 2 receptors comprise an activator that recognizes an antigen present on the surface of normal and tumor cells and a blocker that recognizes a second surface antigen from an allele lost only in tumor cells. HLA LOH has been observed in $\sim 13 \%$ across all solid tumors and up to $33 \%$ of pancreatic cancers. ${ }^{8}$ New technologies have shown higher HLA LOH rates; however, it is unclear whether patients with HLA LOH in their primary tumor tissues are at higher risk for recurrence. BASECAMP-1 is an observational study with key objectives: 1) To determine and identify patients with somatic HLA LOH eligible for Tmod CAR T-cell therapy, and 2) Subsequent leukapheresis and manufacturing feasibility for future Tmod CAR T-cell trials.

Methods BASECAMP-1 (NCT04981119) patient eligibility has 2 parts (figure 2): 1) Patients will be initially screened to identify germline HLA-A*02 heterozygosity by central nextgeneration sequencing (NGS). If HLA-A*02 heterozygosity is confirmed, primary archival tumor tissue will be analyzed by xT-Onco NGS testing ${ }^{9}$ to determine if somatic tumor HLA$\mathrm{A}^{* 02} \mathrm{LOH}$ is present; 2) If the tumor demonstrates HLA$\mathrm{A}^{* 02} \mathrm{LOH}$ and the patient screens eligible, the patient will undergo leukapheresis. Patients enrolled in the study who undergo leukapheresis will be evaluated for safety 7 days post-leukapheresis and followed for relapsed status. Banked $\mathrm{T}$ cells will be available for subsequent autologous Tmod CAR T-cell therapy at the time of relapse.

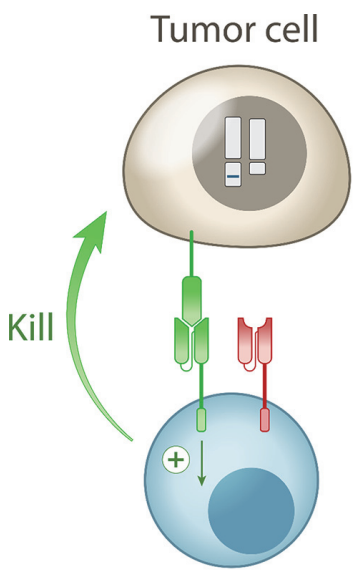

Tmod T cell

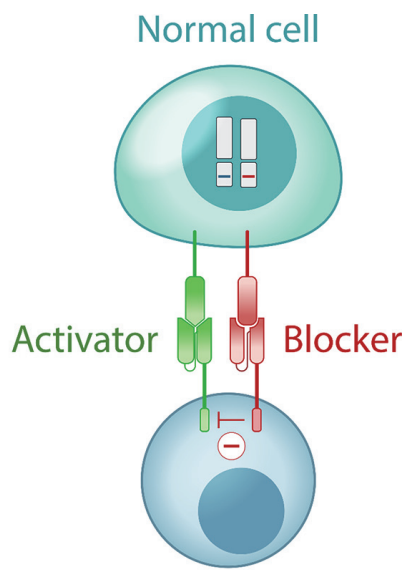

Tmod T cell
Abstract 491 Figure 1 Illustration of the Tmod T cell engaging with tumor cells with somatic loss of HLA-A*02 and with normal cells

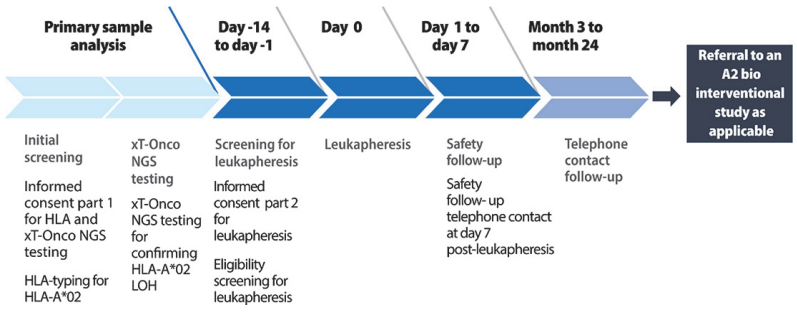

Abstract 491 Figure 2 Study schema. HLA, human leukocyte antigen; LOH, loss of heterozygosity; NGS, next generation sequencing

Trial Registration NCT04981119

\section{REFERENCES}

1. American Cancer Society. Cancer Facts \& Figures 2021. Atlanta: American Cancer Society; 2021

2. Neelapu S, Locke F, Bartlett N, et al. Axicabtagene ciloleucel CAR T-cell therapy in refractory large B-cell lymphoma. N Engl J Med 2017;377(26):2531-2544.

3. Maude S, Laetsch T, Buechner J, et al. Tisagenlecleucel in children and young adults with B-cell lymphoblastic leukemia. N Engl J Med 2018;378(5):439-448.

4. Parkhurst $M$, Yang J, Langan $R$, et al. T cells targeting carcinoembryonic antigen can mediate regression of metastatic colorectal cancer but induce severe transient colitis. Mol Ther 2011:19(3):620-626.

5. Haas AR, Tanyi JL, O’Hara MH, et al. Phase I study of lentiviral-transduced chimeric antigen receptor-modified T cells recognizing mesothelin in advanced solid cancers. Mol Ther. 2019:27(11):1919-1929.

6. Hamburger A, DiAndreth B, Cui J, et al. Engineered T cells directed at tumors with defined allelic loss. Mol Immunol 2020;128:298-310.

7. Hwang M, Mog B, Douglass J, et al. Targeting loss of heterozygosity for cancerspecific immunotherapy. Proc Natl Acad Sci U S A 2021;118(12):e2022410118.

8. The Cancer Genome Atlas (TCGA) Research Network. https://www.cancer.gov/ tcga. Accessed June 2021.

9. Perera J, Mapes B, Lau D, et al. Detection of human leukocyte antigen class I loss of heterozygosity in solid tumor types by next-generation DNA sequencing. $J$ Immunother Cancer 2019;7(suppl 1):103.

http://dx.doi.org/10.1136/jitc-2021-SITC2021.491 